\title{
REVIEW AND ASSESSMENT OF PULSATING HEAT PIPE MECHANISM FOR HIGH HEAT FLUX ELECTRONIC COOLING
}

\author{
G. Karimi ${ }^{\dagger}$, J.R. Culham \\ Microelectronics Heat Transfer Laboratory \\ Department of Mechanical Engineering \\ University of Waterloo \\ Waterloo, Ontario, Canada, N2L 3G1 \\ Phone: (519)888-4586 \\ Fax: (519)746-9141 \\ karimir@mhtlab.uwaterloo.ca \\ rix@mhtlab.uwaterloo.ca
}

\begin{abstract}
Pulsating Heat Pipe (PHP) assisted heat sinks/chips present significantly lower spreading resistance, resulting in a higher heat removal capacity and more uniform temperature in electronic components and provide more packaging flexibilities. Theoretical and experimental investigations have shown that PHPs' heat spreading performance can be as high as that of diamond substrate. This article reviews some of the fundamental mechanisms involved in a PHP operation. The effectiveness of PHPs is presented in terms of heat transfer capacity, flexibilities, and limitations. The performance of a simple heat sink with and without an embedded heat pipe is also evaluated numerically.
\end{abstract}

KEY WORDS: pulsating heat pipe, electronic cooling, heat sink.

\section{NOMENCLATURE}

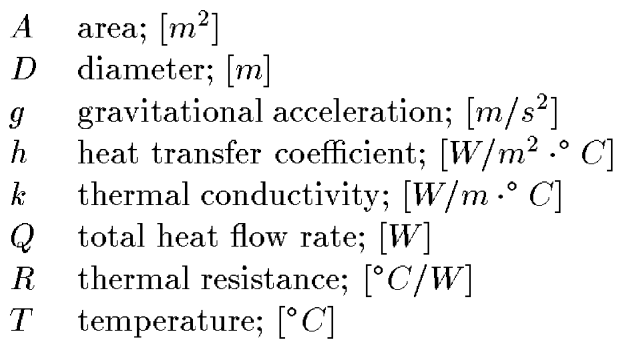

\section{Greek symbols \\ $\Delta$ difference \\ $\rho \quad$ density $\left[\mathrm{kg} / \mathrm{m}^{3}\right]$ \\ $\sigma \quad$ surface tension $[N / m]$}

$\dagger$ Assistant Professor, Department of Chemical Engineering, Shiraz University, Shiraz, Iran

\begin{tabular}{ll}
\multicolumn{2}{l}{ Subscripts } \\
cond & condensation \\
cross & cross section \\
eff & effective \\
evp & evaporation \\
$l$ & liquid \\
$l-v$ & liquid-vapor (two-phase) \\
max & maximum \\
vap & vapor \\
wall & wall
\end{tabular}

\section{INTRODUCTION}

Recent advances in electronics design and manufacturing have resulted in significant increases in heat flux density through a miniaturization of components and a simultaneous increase in power requirements associated with improved product functionality. As a result, thermal management is, and will continue to be one of the highest priority items in the production of high performance microelectronic devices. It will directly impact the cost, reliability, and performance of advanced microelectronic devices.

Thermal management of electronic equipment requires that the heat generated in the chip be removed by natural and/or forced convection and radiation to maintain electronic devices at acceptable temperature levels. To understand the heat transfer mechanisms, a cross-sectional view of a typical flip-chip package with an attached heat sink mounted onto a printed circuit board is shown in Fig. 1. The heat generated on the active side of the chip (i.e. junction) spreads throughout the chip, and is conducted across the thickness of the silicon, then through the heat sink attach material (or thermal interface material), and finally to the heat sink where it is removed by a combination of convection and radiation. On the other side, the chip is attached to the printed circuit board through a packageleads/ceramic-ball-grid-array combination. The simplified 

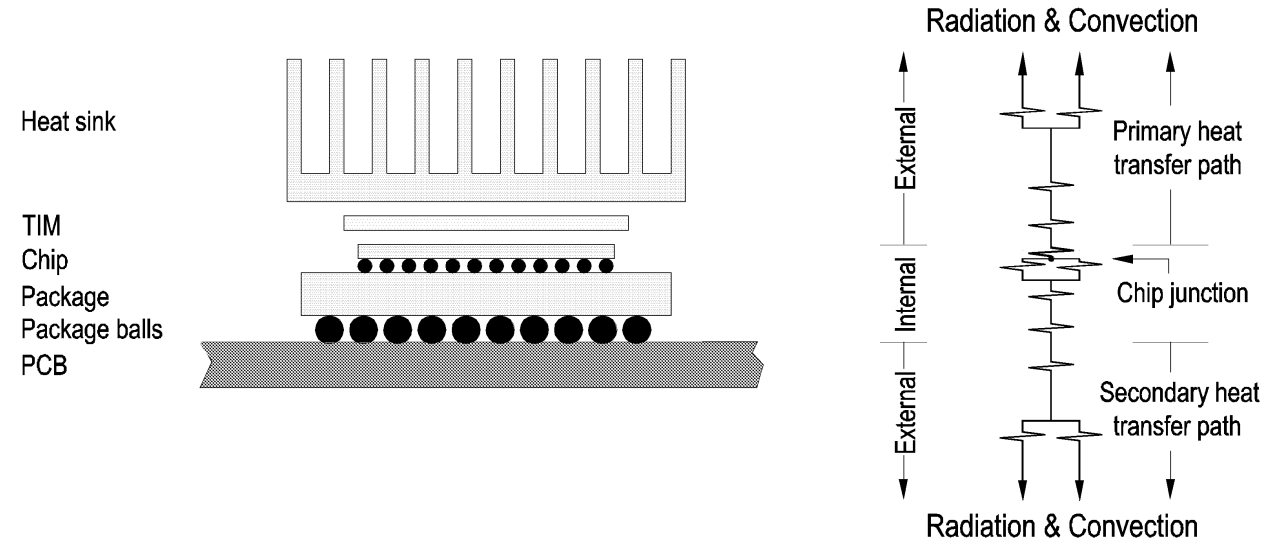

Figure 1: The cross-sectional view of a typical chip and its simplified thermal resistance network model

thermal network is also illustrated in Fig.1.

The heat dissipation phenomenon can be classified into two levels, internal and external. The internal level is primarily concerned with the heat transfer from the silicon chip to highly conductive metallic interconnects and underfill materials as shown in Fig. 1. The external level involves the heat rejection from the package to the ambient. The thermal paths available for this level are either through the printed circuit board or through a heat sinkfan arrangement. Since, the thermal conductivity of the silicon is very high, the heat generated in the junction is removed by connecting a heat sink-fan assembly to the silicon back.

With increasing power levels, the complexity of heat sinks has also increased. New heat sinks are now fabricated with elaborate cross cut extrusions to enhance air flow and their convective capabilities. More effective thermal interface materials are also being used. However, with these efforts to push electrical performance to higher levels, conventional cooling technologies and thermal management are facing growing challenges including; heat fluxes of 10 to $40 \mathrm{~W} / \mathrm{cm}^{2}$, heat loads of $10 \mathrm{~W}$ to $150 \mathrm{~W}$, miniaturization with a tendency towards multi chip modules, long term reliability, and very low costs for consumer market products, among others [1]. This necessitates the development and use of more efficient, nontraditional cooling approaches. Pulsating Heat Pipes (PHPs), characterized by highly effective evaporation and condensation cycles offer an effective thermal conductivity several times greater than traditional heat sinks, present promising alternatives for the removal of high localized heat fluxes and a higher level of temperature uniformity across the electronic components. Compared to other cooling techniques, PHPs do not require mechanical pumps, valves or consume any power and consequently are quieter and more reliable. These features are extremely promising for handling the heat generated in the next generation of microelectronic components or systems.

This article reviews some of the high performance options that are now being employed or developed in thermal management of electronic equipment. Particular attention will be given to the PHPs. The effectiveness of PHPs is presented in terms of heat transfer capacity, flexibilities, and limitations. The performance of a simple heat sink with and without an embedded heat pipe is also evaluated numerically.

\section{HEAT PIPES}

\section{Wicked Heat Pipe}

A traditional heat pipe consists of a vacuum tight envelope, with the internal walls lined with a capillary structure or wick with a working fluid. Figure 2 is a side view of a heat pipe showing the wick and the vapor/liquid flow characteristics. The heat pipe is initially fully evacuated and then back filled with enough working fluid to saturate the wick. The pressure inside the pipe is equal to the saturation pressure associated with the heat pipe temperature. As heat enters the evaporator, equilibrium is perturbed generating vapor at a slightly higher pressure and temperature. The higher pressure causes vapor to flow along the pipe to the condenser end where a slightly lower temperature causes the vapor to condense and release its latent heat of vaporization. The condensed fluid is then pumped back to the evaporator by the capillary forces developed in the wick structure. This continuous cycle can transfer large quantities of heat with very low thermal gradients [2]. The temperature gradient associated with this vapor pressure gradient is typically in the order of a couple of degrees Celsius [3].

Although the first wicked heat pipe was invented in 1963 , it was in the early 1990 's that the heat pipe started to be used in high volume consumer electronics. Today, heat pipes are an accepted thermal management tool used in many applications including workstations, mainframes, power supplies, and heat exchangers because of their performance and reliability. Almost every current notebook computer uses a heat pipe to transfer heat from the CPU 


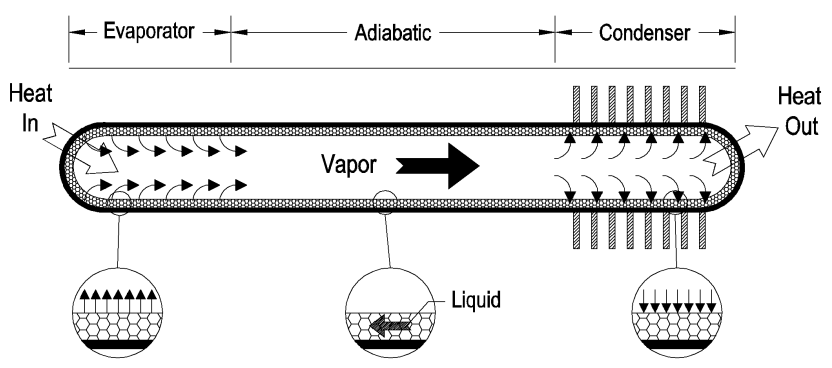

Figure 2: The cross-sectional view of a typical wicked heat pipe

to the EMI shield of the keyboard and then by natural convection and radiation to the surrounding. This type of passive design has been a very effective thermal management technique for low power CPU's [4].

Although a variety of designs are available for classical heat pipes, the growing demands on the thermal management have frequently shown the limitations and shortcomings of these conventional designs including geometric (e.g size) and operating (e.g. orientation, capillary, boiling, and entrainment among others) limits [5]. This has led to the evolution of novel concepts fitting to the need of the future electronic industry demands.

\section{Pulsating Heat Pipe}

A meandering tube PHP, as proposed by Akachi [6] consists of a metallic tube of capillary dimensions wound in a serpentine manner and joined end to end. It is first evacuated and then filled partially with a working fluid, which distributes itself naturally in the form of liquid-vapor plugs and slugs inside the capillary tube as shown in Fig. 3. One end of this bundle of tubes receives heat (evaporator section) transferring it to the other end (condenser section) by a pulsating action of the liquid-vapor system. There may exist an optional adiabatic zone in between. A PHP is essentially a non-equilibrium heat transfer device whose performance success depends primarily on the continuous maintenance of these non-equilibrium conditions within the system. The liquid-vapor slug transport is due to thermally driven pressure pulsations in the respective tubes as described below.

Operations: Consider a case when a PHP is isothermal throughout. Under this condition, the liquid and vapor phases in the PHP exist in equilibrium at a saturation pressure corresponding to the fixed isothermal temperature. In Fig. 4, the average thermodynamic state of the heat pipe can be represented by point A . During operation, a temperature gradient prevails between the evaporator and the condenser, causing non-equilibrium pressure conditions. The heat transfer to the evaporator causes the bubbles in the evaporator to grow continuously and tries to move point $\mathrm{A}$ to point $\mathrm{B}$ at a higher pressure/temperature and a larger quality. This pushes the liquid column toward the low-temperature end (condenser). Simultaneously, the

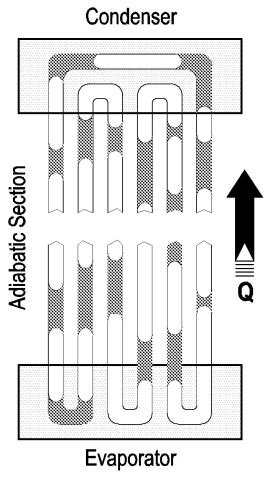

Figure 3: The cross-sectional view of a typical PHP

condensation at the other end will further enhance the pressure difference between the two ends, and point $\mathrm{A}$ is forced to move to point $\mathrm{C}$ at a lower pressure/temperature and a smaller quality. In this way a non-equilibrium state is formed between the driving thermal potentials and the system in return attempts to equalize the internal pressure. Because of the inter-connection of the tube(s), the motion of the liquid slugs and vapor bubbles at one section of the tube towards the condenser also leads to the motion of slugs and bubbles in the other section towards the hightemperature (evaporator) in the next section. This works as a restoring force. The inter-play between the driving force and restoring force leads to oscillation of the vapor bubbles and liquid slugs in the axial direction. Further, inherent perturbations present in real systems augment pressure fluctuations in the system.

Unlike conventional heat pipes, no steady-state pressure equilibrium can be achieved for an operating PHP. The frequency and amplitude of the oscillations are expected to depend on the heat flow rate and the mass fraction of the liquid in the tube. Through these oscillations, heat which is supplied by the heat source at the evaporator is carried to the condenser and is removed by a heat sink.

Performance: The performance of a PHP depends on its structure, shape, material, and length. A major criterion for thermal performance is the temperature drop along the heat pipe for a required heat load. Figure 5 shows thermal resistance components of a PHP. The overall thermal resistance is composed of five components from the evaporating end to the condensing end: two conductive thermal resistances in the wall, $R_{w a l l}$, thermal resistances due to the evaporation and condensation at the evaporator and condenser, $R_{e v p}$, and $R_{\text {cond }}$, respectively, and a thermal resistance along the heat pipe length, $R_{l-v}$. In the case of a wicked heat pipe additional thermal resistances need to be added into the wall resistance due to the presence of the wick.

The heat transfer capacity of a PHP, $Q$, can be calculated through the following equation: 


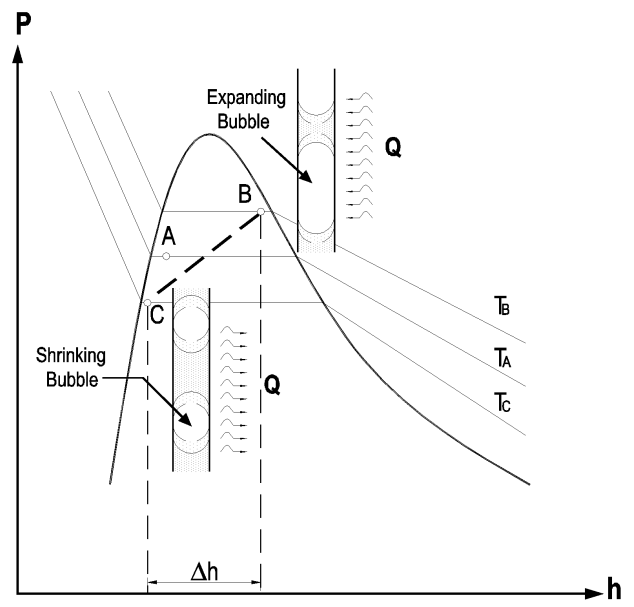

Figure 4: Pressure-enthalpy diagram for a PHP

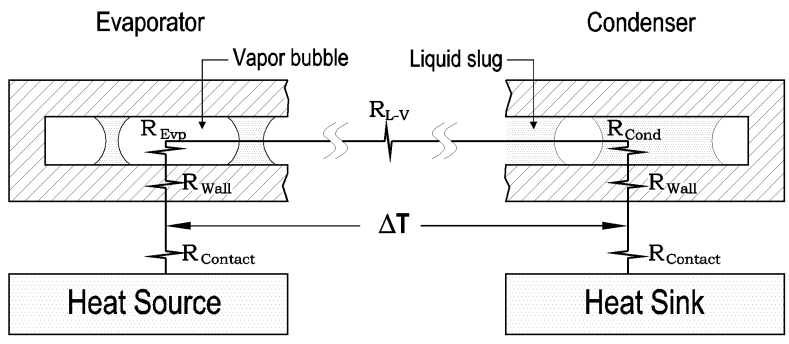

Figure 5: Components of the thermal resistances of a typical PHP

$$
Q=\frac{\Delta T}{2 R_{w \text { all }}+R_{e v p}+R_{l-v}+R_{\text {cond }}}=\frac{\Delta T}{\frac{L_{c f f}}{k_{c f f f \cdot A_{c r o s s}}}}
$$

where $\Delta T$ is the overall temperature difference along the heat pipe, $k_{\text {eff }}$ and $L_{\text {eff }}$ are the effective thermal conductivity and length, and $A_{\text {cross }}$ is the cross sectional area of the PHP.

A properly mounted PHP introduces small thermal contact resistances. The conductive thermal resistance in the wall is also negligible. For instance, a $1 \mathrm{~mm}$ thick copper wall introduces about $\left(2.6 \times 10^{-6} / A\right)^{\circ} \mathrm{C} / W$ resistance to the heat flow where $A$ is the heating area $\left[\mathrm{m}^{2}\right]$. Both contact and conductive resistances are almost independent of the heat pipe operating temperatures. Therefore, the thermal resistances due to the evaporation in the evaporator, $R_{\text {evp }}$, two-phase flow along the heat pipe, $R_{l-v}$, and the condensation in the condenser, $R_{\text {cond }}$, are critical to the performance of the heat pipe.

Under normal operation, a continuous flow boiling associated with vigorous bubble growth and slug flow takes place in the evaporator section of the heat pipe. The two phase flow continues its way towards the heat pipe condenser where bubbles are collapsed while giving up their latent heat of vaporization.

Extensive research has been conducted in recent years to measure flow boiling heat transfer coefficient in mini channels $[7,8,9,10,11]$, among others. The reported experimental data covers a wide range of channel diameter (from $400 \mu \mathrm{m}$ to $2.97 \mathrm{~mm}$ ), mass flux (from 50 to 600 $\mathrm{kg} / \mathrm{m}^{2} . \mathrm{s}$ ), and heat flux (from 5 to $600 \mathrm{~kW} / \mathrm{m}^{2}$ ). The experimental measurements have shown that the heat transfer coefficient can either increase, remain constant, or decrease with quality depending on the boiling number and liquid-to-vapor density ratio. The flow boiling heat transfer coefficients reported varied between 1000 to $6500 \mathrm{~W} / \mathrm{m}^{2} \cdot{ }^{\circ} \mathrm{C}$ [12]. Having considered these data, the corresponding thermal resistance in the heat pipe evaporator can be estimated to be between $(0.001 / A)$ and $\left(1.54 \times 10^{-4} / A\right){ }^{\circ} \mathrm{C} / W$, respectively. A similar heat transfer coefficient range for the flow condensation in the evaporator seems to be reasonable.

The liquid-vapor thermal resistance along the PHP, $R_{l-v}$, is a function of the pressure/temperature drop from evaporator to condenser. For a wicked heat pipe, $R_{l-v}$ accounts for only $5 \%$ of the total resistance and is almost independent of the heat pipe length and the number of bends [13]. This near isothermal nature regardless of length is the principal benefit that the heat pipe offers to the system thermal design.

Having considered the range of thermal resistances from the heat source to the heat sink in an operating PHP, the total thermal resistance for a typical PHP is estimated to be in the range of $\left(3.29 \times 10^{-4} / A\right)$ and $\left(2.10 \times 10^{-3} / A\right)$ ${ }^{\circ} \mathrm{C} / W$.

As mentioned earlier, heat pipes are heat transfer devices and must be coupled to other components (such as a heat sink) to dissipate heat through natural or forced convection. In other words, heat pipes add high thermal 
Table 1: Estimated effective thermal conductivities as a function of PHP dimensions

\begin{tabular}{|c|c|c|c|}
\hline \hline $\begin{array}{c}A_{c} \text { ross } \\
{\left[\mathrm{cm}^{2}\right]}\end{array}$ & $\begin{array}{c}A_{\text {evp }}=A_{\text {cond }} \\
{\left[\mathrm{cm}^{2}\right]}\end{array}$ & $\begin{array}{c}k_{\text {eff,min }} \\
{\left[W / \mathrm{m}^{2} \cdot{ }^{\circ} \mathrm{C}\right]}\end{array}$ & $\begin{array}{c}k_{\text {eff, max }} \\
{\left[\mathrm{W} / \mathrm{m}^{2} \cdot{ }^{\circ} \mathrm{C}\right]}\end{array}$ \\
\hline \hline 0.3 & 9 & 2,857 & 18,237 \\
\hline 0.4 & 16 & 3,809 & 24,316 \\
\hline 0.6 & 9 & 1,428 & 9,118 \\
\hline 0.9 & 16 & 1,905 & 12,158 \\
\hline \hline
\end{tabular}

conductivity thermal links permitting the heat sink to be located remote from the heat source. Obviously, the higher the effective thermal conductivity, a more efficient thermal link is obtained. Effective thermal conductivity of 60 times better than that of copper has been reported for wicked heat pipes [13]. For a PHP of estimated thermal resistances just mentioned above, the effective thermal conductivity can be estimated using Eq. 1. Table 1 lists the estimated minimum and maximum effective thermal conductivities for a $20 \mathrm{~cm}$ long PHP having different heating/cooling and cross sectional areas. As seen from this table, a typical PHP is capable of providing a thermal link between the heat source and a heat sink with effective thermal conductivities as high as $24 \mathrm{~kW} / \mathrm{m} \cdot{ }^{\circ} \mathrm{C}$, some 10 times better than diamond.

Working Fluid: A first consideration in the selection of a suitable working fluid is the operating vapor temperature range. Within the approximate temperature band, $\left(50\right.$ to $150{ }^{\circ} \mathrm{C}$ ) several possible working fluids may exist. A variety of characteristics must be examined in order to determine the most acceptable of these fluids for the application considered. The prime requirements are: compatibility with the heat pipe material(s), thermal stability, wettability, reasonable vapor pressure, high latent heat and thermal conductivity, low liquid and vapor viscosities, and acceptable freezing point.

For most commercial electronics cooling applications, the thermodynamic attributes of water make it better than any other fluids for both wicked and pulsating heat pipes. Its high latent heat spreads more heat with less fluid flow. This results in low pressure drops and high power throughput. Its high thermal conductivity minimizes the $\Delta T$ associated with conduction through the wick in case of wicked heat pipes or through the two-phase flow in the PHP. Water is also a safe substance.

Although water's high surface tension allows it to generate a large capillary force and allows the wicked heat pipe to operate in any orientation, it may have adverse effects on the operation of a PHP. In other words, the high surface tension may cause additional friction and hinder the two-phase flow oscillations in the PHP. Methanol with a lower surface tension (about 1/3rd of water) is a good substitute particularly if the heat pipe is used for sub 0 ${ }^{\circ} \mathrm{C}$ applications.
Hydraulic Diameter As indicated in Eq. 1, a larger hydraulic diameter results in lower wall thermal resistances and increases the effective thermal conductivity for both wicked and pulsating heat pipes. However, the internal diameter is a parameter which necessarily affects the very definition of a pulsating heat pipe. A theoretical maximum tolerable inner diameter, $D_{M a x}$, of a PHP capillary tube was derived based on the balance of capillary and gravity forces by Akachi and Polasek [14] as:

$$
D_{\text {max }}=2 \sqrt{\frac{\sigma}{g\left(\rho_{l}-\rho_{\text {vap }}\right)}}
$$

where $\sigma, g$, and $\rho$ are surface tension, gravitational acceleration, and density, respectively. If $D<D_{\max }$ surface tension forces tend to dominate and stable liquid slugs are formed. As the PHP tube diameter increases beyond the $D_{\max }$, the surface tension is reduced and all the working fluid will tend to stratify by gravity and the heat pipe will stop functioning as a PHP, and the device may operate as an interconnected array of two-phase thermosyphons.

Filled Ratio: Filled ratio is the fraction (by volume) of the heat pipe which is initially filled with the liquid. There are two operational filled ratio limits. At $0 \%$ filled ratio, a heat pipe structure with only bare tubes and no working fluid, is a pure conduction mode heat transfer device with a very high undesirable thermal resistance. A $100 \%$ fully filled heat pipe is identical in operation to a singlephase thermosyphon. The pulsating effect is nonexistent however, substantial heat can be transferred due to liquid circulation in the tubes by thermally induced buoyancy. The thermosyphon action is maximum for a vertical heat pipe and stops for a horizontal heat pipe and heat transfer takes place purely by axial conduction.

If the heat transfer rate has to be augmented, a logical step is to introduce a two-phase flow regime rather than a single phase system. By partially filling the PHP, it is expected to achieve a latent heat advantage due to the evaporation and condensation of bubbles. Also, the temperature gradient between heater and cooler coupled with bubble growth and collapse will generate self-sustaining pressure perturbations, as explained earlier, causing liquid plug transport and thus sensible heat transfer. This feature is also expected to make the PHP performance to be orientation independent.

In a PHP there are three distinct operational regions: (a) Near 100\% filled ratio: In this case there are very few bubbles present which are insufficient to generate the required perturbations. Even the buoyancy induced liquid circulation, which was present at $100 \%$ filled ratio, gets hindered due to additional surface tension generated friction of the bubbles. Thus the performance of the devise is seriously hampered. (b) Near $0 \%$ filled ratio: In this mode there is very little liquid to form enough distinct slugs and there is a tendency towards dry-out of the evaporator. Therfore, the PHP introduces an undesirable thermal resistance. (c) PHP true working range: Between 
about 20 to $70 \%$ filled ratio, the PHP operates as a true pulsating device. The exact range will differ for different working fluids, operating parameters and construction. More the bubbles are (lower filled ratio), more intense are the pulsations but simultaneously there is less liquid mass for sensible heat transfer. On the other hand, fewer bubbles at highly filled ratios cause less perturbations and the bubble pumping action is reduced thereby lowering the performance. Thus an optimum fill charge exists [15].

Operating Temperatures: Heat pipes can be designed to operate over a very broad range of temperatures from cryogenic applications to high temperature applications. In electronic cooling applications where it is desirable to maintain junction temperatures below $125-150{ }^{\circ} \mathrm{C}$, copper/water heat pipes are typically used. Copper/methanol heat pipes are used if the application requires heat pipe operation below $0^{\circ} \mathrm{C}$.

Reliability: If the heat pipe material(s) are compatible with the working fluid, they can be expected to provide highly reliable heat transfer performance within their operating limits for years. Heat pipes have been widely used in military applications which have very high reliability standards. The copper/water materials used in heat pipes employed in notebooks technology have been life tested in Thermacore Inc. for over 17 years now. When properly manufactured, copper/water heat pipes can reasonably be expected to operate for over 30 years, well beyond the life/usefulness expected of the electronics heat pipes are intended to cool [13].

Future Application: Wicked heat pipes are currently used in a wide range of microelectronic markets, ranging from high-end workstation applications to power electronic cooling applications. Heat pipe assisted heat sink systems are still undergoing further developments and their applications in electronic cooling are limited primarily due to their costs.

As the chips in lower-end devices get smaller and operate hotter, thermal management is becoming one of the major considerations in creating tomorrow's more compact, complex micro devices. Traditionally microchip substrates are made of fiberglass-epoxy-type materials loaded with copper interconnects that help conduct heat into the substrate and away from the microchip. Other designs include supplementary heat spreaders of solid copper or other conductive materials within the substrate. In the recent past, highly conductive diamond and diamond-coated substrates have been developed, but their use is drastically limited by their high cost.

To solve the heat-buildup problem in microelectronic devices, future electronic devices could benefit from embedded PHPs in their chips or PHP assisted heat sinks to help carry some heat away from the hottest circuitry and transfer it to other structural elements. Micro PHPs can be built directly within the substrate material using microfabrication technology to carry heat away from the chip much more efficiently than conduction through solid copper or transport through larger heat pipes on the substrate surface and thus allow for greater heat reduction and improved temperature control. Also, mini PHPs can be either embedded or directly fabricated inside the heat sink bases to enhance their heat transfer capacities.

Modeling: The invention of PHP has motivated many researchers to develop physical models underlying its operation. The complexity of thermo-fluidic transport phenomena in the PHPs is so overwhelming that a comprehensive theory and reliable data or tools for the design of PHPs according to a given microelectronics cooling requirement, are still an unrealized task. The various published approaches to model the physical processes within a PHP are mostly based on very rough assumptions and simplifications. Currently, PHPs are simulated as simple mass-spring combinations where liquid plugs (masses) and vapor bubbles (acting as springs) are interacting with a distinct interface (see for example $[16,17]$ ).

Case Study: In this section, the effectiveness of a PHP is studied by embedding it into a heat sink base. In Fig. $6,20 \mathrm{~W}$ heat is generated in a $1 \mathrm{~cm}^{2}$ silicon chip $(2 \mathrm{~mm}$ thick) which is then conducted to the base of $16 \mathrm{~cm}^{2}$ aluminum heat sink ( $3 \mathrm{~mm}$ thick) before it is dissipated into the ambient air. The lower surface is intentionally insulated. The cross sectional area of PHP is considered to be $1 \mathrm{~mm}^{2}$ which extends from the chip area (e.g evaporator) to the sides (e.g. condenser). A range of effective thermal conductivities assigned to the embedded PHP.

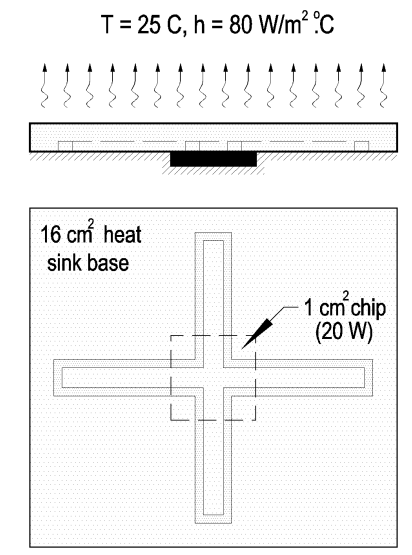

Figure 6: A representative of embedded PHP heat sink

The thermal simulations were conducted using I-DEAS 10 and the TMG package as the simulation platform. Figure 7 illustrates the temperature distributions in the PHP assisted heat sink under steady-state conditions. The upper plots are for the heat sink surface and the lower graphs are for the lower surface where the chip is located. As seen from the figure, the embedded heat pipe could reduce the chip temperature by increasing the effective thermal conductivity of the heat sink. However, with the current 
Table 2: Estimated spreading resistances for a PHP assisted heat sink as a function of PHP effective thermal conductivity

\begin{tabular}{|c|c|c|c|c|}
\hline Case & $\begin{array}{c}k_{e} f f \\
{\left[W / m^{2} \cdot{ }^{\circ} C\right]}\end{array}$ & $\begin{array}{l}\bar{T}_{\text {chip }} \\
{\left[{ }^{\circ} \mathrm{C}\right]}\end{array}$ & $\begin{array}{c}\overline{\bar{T}}_{\operatorname{sink}} \\
{\left[{ }^{\circ} \mathrm{C}\right]}\end{array}$ & $\begin{array}{c}\overline{\bar{R}} \\
{\left[{ }^{\circ} \mathrm{C} / W\right]}\end{array}$ \\
\hline $\mathrm{a}($ no $\mathrm{PHP})$ & 200 & 152.6 & 146.9 & 0.29 \\
\hline $\mathrm{b}$ & 1000 & 151.5 & 146.8 & 0.23 \\
\hline $\mathrm{c}$ & 2000 & 150.8 & 146.7 & 0.20 \\
\hline $\mathrm{d}$ & 10000 & 149.2 & 146.5 & 0.13 \\
\hline
\end{tabular}

arrangement, both the chip and heat sink temperatures are fairly high. In practice, there are other factors which lower the chip temperature to an acceptable range. The heat sink is equipped with fins which increases the surface area significantly. Also, part of the heat is dissipated in the other direction. Also, the current arrangement of PHP is chosen for simplicity. In reality, the PHP could cover a larger area of the heat sink base resulting in a lower thermal resistance.

For the present case, the spreading resistance from the chip to the heat sink base is calculated as a function of effective PHP thermal conductivity. Table 2 lists the spreading resistances for different cases. As seen from the table, the spreading resistance of a unasssited heat sink could drop by at least $50 \%$ when a simple PHP is embedded inside its base.

\section{SUMMARY}

Application of PHPs in thermal management of electronic equipment was reviewed. The effectiveness of a typical operating PHP was estimated using an equivalent thermal resistance network. The PHP effective thermal conductivity could be as high as $24 \mathrm{~kW} / \mathrm{m} \cdot{ }^{\circ} \mathrm{C}$. Numerical simulation for a PHP assisted heat sink has shown that the spreading resistance could reduce by at least $50 \%$.

Acknowledgments The authors would like to thank Mr. Edward Chan for the numerical simulation of the PHP assisted heat sink.

\section{References}

[1] International technology roadmap for semiconductors, 2002 update. http://public.itrs.net/

[2] Garner, S.D., 1996, "Heat Pipes For Electronics Cooling Applications", Electronics Cooling, Vol 2, No. 3.

[3] Faghri, A., Heat Pipe Science and technology, Taylor \& Francis, Washington D.C., 1995.

[4] Ali, A, Dehoff, R., \& Grubb, K, 2000, "Advanced Heat Pipe Thermal Solutions for Higher Power Note- book Computers", 2000 Pan Pacific Symposium Conference.

[5] Nishio, S., 2000, "Attempts to Apply Micro Heat Transfer to Thermal Management". Int. Conf. on Heat Transfer and Transport Phenomena in Microscale, Banff, Canada.

[6] Akachi, H, 1990, "Structure of a Heat Pipe", US Patent, No 4,921,041.

[7] Wambsganss, M.W., et al., 1993, "Boiling Heat Transfer in a Horizontal Small-Diameter Tube". Journal of Heat Transfer, vol. 115, pp. 963-972.

[8] Ravigururajan, T.S., et al., 1996, "Effects of Heat Flux on Two-Phase Flow Characteristics of Refrigerant Flows in a Micro- Channel Heat Exchanger". Proceedings of National Heat Transfer Conference HTD329 , pp. 167-178, 1996.

[9] Yan, Y., and T. Lin, 1998, "Evaporation Heat Transfer and Pressure Drop of Refrigerant R-134a in a Small Pipe". International Journal of heat and Mass Transfer, vol. 41, pp. 4183-4194, 1998.

[10] Kamidis, D.E., and T.S. Ravigururajan, 1999, "Single and Two-Phase Refrigerant Flow in Mini-Channels", Proc. of 33rd National Heat Transfer Conference, Albuquerque, NM, Aug 15-17, 1999.

[11] Lin, S., 1999, "Two-Phase Evaporation in a $1 \mathrm{~mm}$ Diameter Tube", Proc. of 6th UK Heat Transfer Conference, 1999.

[12] Kandlikar, S.G., and Steinke, M.E., 2002, "Flow Boiling Heat Transfer Coefficient in MinichannelsCorrelation and Trends", Proc. of 12th Int. Heat Transfer Conference, Grenoble, France, Paper \# 1178.

[13] Xie, H. Aghazadeh, M. and Toth. J., 1995, "The use of heat pipes in the cooling of portables with high power packages-A case study with the Pentium processorbased notebooks and sub-notebooks". Proc. of the 1995 Electronic Components and Technology Conference (ECTC), pp. 906-913, May 21-24, 1995.

[14] Akachi H., Polasek, F. and Stulc P., 1996, "Pulsating Heat Pipes", Proc. 5th Int. Heat Pipe Symp., Melbourne, Australia, pp. 208-217, 1996.

[15] Khandekar, S, Dollinger, N, and Groll, M., 2003, "Understanding Operational Regimes of Closed Loop Pulsating Heat Pipes: An Experimental Study", Applied Thermal Engineering, Vol. 23, No. 6, pp. 707-719

[16] Ma, H.B., Hanlon, M.A., and Chen, C.L., 2001, “An Investigation of Oscillation Motions in a Pulsating Heat Pipe". Proc. of 35th National Heat Transfer Conference, Anaheim, California, June 10-12, 2001.

[17] Wong, T.N., Tong, B.Y. Lim, S.M., 1999, "Theoretical Modeling of Pulsating Heat Pipes". 11th Int. Heat Pipe Conf., Tokyo, Japan, Sept. 1999. 
(a)
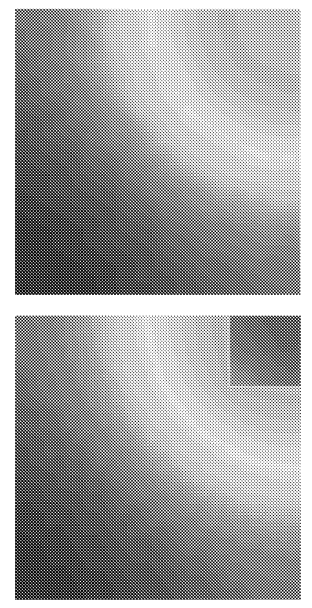

(b)
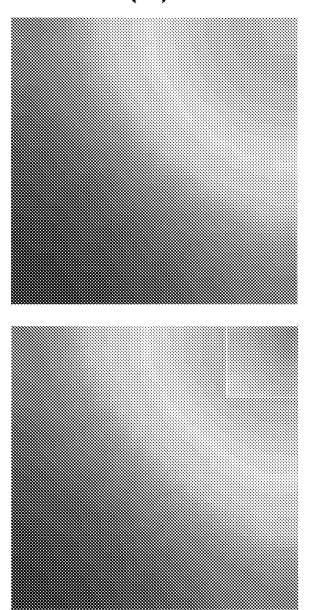

(c)
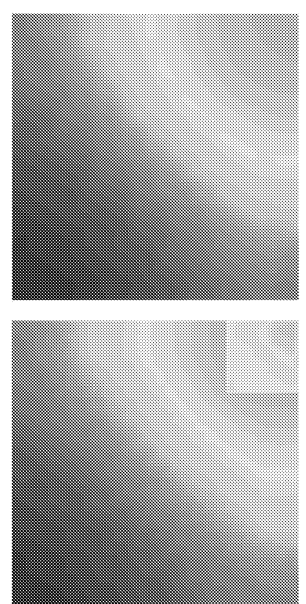

(d)

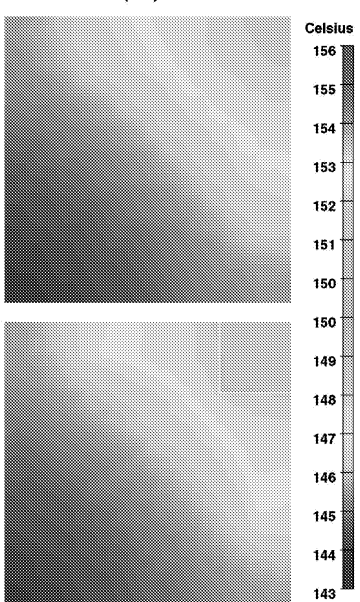

Figure 7: Steady-state temperature distribution in a PHP assisted heat sink. (a) $k_{P H P}=200$, (b) $k_{P H P}=1000$, (c) $k_{P H P}=2,000$, and $(\mathrm{d}) k_{P H P}=10,000 \mathrm{~W} / \mathrm{m} \cdot{ }^{\circ} \mathrm{C}$. 